ANNALES

POLONICI MATHEMATICI

$93.1(2008)$

\title{
Positive periodic solutions of functional differential equations with infinite delay
}

\author{
by Changxiu Song (Guangzhou)
}

Abstract. The author applies a generalized Leggett-Williams fixed point theorem to the study of the nonlinear functional differential equation

$$
x^{\prime}(t)=-a(t, x(t)) x(t)+f\left(t, x_{t}\right) .
$$

Sufficient conditions are established for the existence of multiple positive periodic solutions.

1. Introduction. In this paper, we are concerned with the functional differential equation

$$
x^{\prime}(t)=-a(t, x(t)) x(t)+f\left(t, x_{t}\right),
$$

where

- $a \in C(\mathbb{R} \times \mathbb{R}, \mathbb{R})$ with $a(t+\omega, x) \equiv a(t, x)$;

- $\forall t \in \mathbb{R}, x_{t}=x_{t}(\theta)=x(t+\theta),-\infty<\theta \leq 0$; we assume $x_{t} \in C$, where $C=C((-\infty, 0], \mathbb{R})$ is a Banach space with the norm $\|\varphi\|_{C}=$ $\max _{\theta \in(-\infty, 0]}|\varphi(\theta)|$;

- $f \in C\left(\mathbb{R} \times C^{+}, \mathbb{R}\right)$ with $f(t+\omega, \varphi) \equiv f(t, \varphi), \varphi \in C^{+}$, where $C^{+}=$ $\{\varphi \in C: \varphi(\theta) \geq 0, \theta \in(-\infty, 0]\}$;

- $\omega>0$.

We make the following assumptions:

$\left(\mathrm{H}_{1}\right)$ there exist $\omega$-periodic functions $a_{1}, a_{2} \in C(\mathbb{R}, \mathbb{R})$ satisfying

$$
a_{1}(t) \leq a(t, x) \leq a_{2}(t), \quad \int_{0}^{\omega} a_{1}(t) d t>0 ;
$$

2000 Mathematics Subject Classification: Primary 34C25.

Key words and phrases: functional differential equation, positive periodic solutions, cone, fixed point theorem.

Supported by grant 10571064 from NNSF of China, and by a grant from NSF of Guangdong. 
$\left(\mathrm{H}_{2}\right) f(t, \varphi)$ maps bounded sets into bounded sets and is a nonnegative continuous function defined on $\mathbb{R} \times C^{+}$.

Our purpose is to prove that (1.1) has multiple positive periodic solutions by using a generalized Leggett-Williams fixed point theorem. For the biological senses of $(1.1)$, we refer to $[2,3,6]$.

From (1.1) we obtain

$$
\left[x(t) \exp \left(\int_{0}^{t} a(\tau, x(\tau)) d \tau\right)\right]^{\prime}=\exp \left(\int_{0}^{t} a(\tau, x(\tau)) d \tau\right) f\left(t, x_{t}\right) .
$$

After integration from $t$ to $t+\omega$, we obtain

$$
x(t)=\int_{t}^{t+\omega} G(t, s) f\left(s, x_{s}\right) d s
$$

where

$$
G(t, s)=\frac{\exp \left(\int_{t}^{s} a(\tau, x(\tau)) d \tau\right)}{\exp \left(\int_{0}^{\omega} a(\tau, x(\tau)) d \tau\right)-1}
$$

Let

$$
\begin{gathered}
M_{1}=\inf _{0 \leq t \leq s \leq \omega} \exp \left(\int_{t}^{s} a_{1}(\tau) d \tau\right), \quad M_{2}=\sup _{0 \leq t \leq s \leq \omega} \exp \left(\int_{t}^{s} a_{2}(\tau) d \tau\right), \\
k_{1}=\exp \left(\int_{0}^{\omega} a_{1}(\tau) d \tau\right), \quad k_{2}=\exp \left(\int_{0}^{\omega} a_{2}(\tau) d \tau\right), \quad \delta=\frac{M_{1}\left(k_{1}-1\right)}{M_{2}\left(k_{2}-1\right)} ;
\end{gathered}
$$

we know that $0<\delta<1$. Furthermore, we have from $\left(\mathrm{H}_{1}\right)$ and (1.4)

$$
\frac{M_{1}}{k_{2}-1} \leq G(t, s) \leq \frac{M_{2}}{k_{1}-1} \text {. }
$$

Now, let $X$ be the set of all real $\omega$-periodic continuous functions, endowed with the usual linear structure as well as the norm $\|x\|=\sup _{t \in[0, \omega]}|x(t)|$. It is a Banach space with a cone $P=\left\{x \in X: x(t) \geq \delta\|x\|, x(t) \exp \left(\int_{0}^{t} a_{1}(\tau) d \tau\right)\right.$ is nondecreasing on $\left.[0, \omega]\right\}$.

Furthermore, for all $x \in X$, we have

$$
\|x\|=\left\|x_{t}\right\|_{C} \quad \text { for each } t \in[0, \omega],
$$

and for all $x \in P$, we deduce that $x(t) \exp \left(\int_{0}^{t} a_{2}(\tau) d \tau\right)$ is nondecreasing on $[0, \omega]$.

Define $T: P \rightarrow X$ as

$$
T x(t)=\int_{t}^{t+\omega} G(t, s) f\left(s, x_{s}\right) d s .
$$

Similar to the proofs of Lemmas 2.2 and 2.4 in [4], one can easily show 
Lemma 1.1. $T: P \rightarrow P$ is well defined and completely continuous.

One can easily see that $x$ is a positive solution of (1.1) if and only if $x$ is a fixed point of $T$ on $P$.

For convenience, we present a generalized Leggett-Williams fixed point theorem due to Avery and Henderson [1]. Let

$$
\begin{aligned}
P(\delta, e) & =\{x \in P: \delta(x)<e\}, \\
\partial P(\delta, e) & =\{x \in P: \delta(x)=e\}, \\
\overline{P(\delta, e)} & =\{x \in P: \delta(x) \leq e\} .
\end{aligned}
$$

Lemma 1.2. Let $X$ be a real Banach space, $P$ a cone of $X, \gamma$ and $\alpha$ two nonnegative increasing continuous function on $P$, and $\theta$ a nonnegative continuous function on $P$ with $\theta(0)=0$ such that there are positive numbers $c$ and $M$ satisfying

$$
\gamma(x) \leq \theta(x) \leq \alpha(x), \quad\|x\| \leq M \gamma(x) \quad \text { for } x \in \overline{P(\gamma, c)} .
$$

Moreover, assume that $T: \overline{P(\gamma, c)} \rightarrow P$ is completely continuous and there are positive numbers $0<a<b<c$ such that

$$
\theta(\lambda x) \leq \lambda \theta(x) \quad \text { for all } \lambda \in[0,1] \text { and } x \in \partial P(\theta, b),
$$

and

(i) $\gamma(T x)>c$ for $x \in \partial P(\gamma, c)$;

(ii) $\theta(T x)<b$ for $x \in \partial P(\theta, b)$;

(iii) $\alpha(T x)>a$ and $P(\alpha, a) \neq \emptyset$ for $x \in \partial P(\alpha, a)$.

Then $T$ has at least two fixed points $x_{1}, x_{2} \in \overline{P(\gamma, c)}$ satisfying

$$
a<\alpha\left(x_{1}\right), \quad \theta\left(x_{1}\right)<b, \quad b<\theta\left(x_{2}\right), \quad \gamma\left(x_{2}\right)<c .
$$

The following lemma is similar to Lemma 1.2.

LEMMA 1.3. The conclusion of Lemma 1.2 still holds if we replace (i)-(iii) there by

(i) $\gamma(T x)<c$ for $x \in \partial P(\gamma, c)$;

(ii) $\theta(T x)>b$ for $x \in \partial P(\theta, b)$;

(iii) $\alpha(T x)<a$ and $P(\alpha, a) \neq \emptyset$ for $x \in \partial P(\alpha, a)$.

LEMma $1.4([5])$. Let $P$ be a cone of a real Banach space $X, \Omega$ a bounded open subset of $X$, and $0 \in \Omega$. Moreover, assume that $T: \overline{P \cap \Omega} \rightarrow P$ is completely continuous and satisfies

$$
T x=\lambda x \text { for some } x \in P \cap \partial \Omega \Rightarrow \lambda<1 \text {. }
$$

Then

$$
i(T, P \cap \Omega, P)=1 \text {. }
$$


REMARK 1.1. We know that $\Omega=P(\alpha, a)$ in Lemma 1.3 is a bounded open subset of $X$, and by (iii) of Lemma 1.3, we have

$$
T x<x \quad \text { for all } x \in P \cap \partial \Omega=\partial P(\alpha, a) .
$$

From Lemma 1.3, Lemma 1.4 and Remark 1.1, we have the following result.

Lemma 1.5. Let the conditions of Lemma 1.3 hold. Furthermore, assume $\theta \in P(\alpha, a)$. Then $T$ has at least three fixed points $x_{1}, x_{2}, x_{3} \in \overline{P(\gamma, c)}$ satisfying

$$
\alpha\left(x_{1}\right)<a, \quad a<\alpha\left(x_{2}\right), \quad \omega\left(x_{2}\right)<b, \quad b<\omega\left(x_{3}\right), \quad \gamma\left(x_{3}\right)<c .
$$

2. Main results. Fix $0 \leq \eta<l \leq \omega$ and define nonnegative, increasing, continuous functions $\gamma, \theta$, and $\alpha$ on $P$ by

$$
\begin{gathered}
\gamma(x)=\min _{\eta \leq t \leq l} e^{\int_{0}^{t} a_{1}(\tau) d \tau} x(t)=x(\eta) e^{\int_{0}^{\eta} a_{1}(\tau) d \tau}, \\
\theta(x)=\max _{0 \leq t \leq \eta} e_{0}^{t_{0}^{t} a_{1}(\tau) d \tau} x(t)=x(\eta) e^{\int_{0}^{\eta} a_{1}(\tau) d \tau}, \\
\alpha(x)=\min _{l \leq t \leq \omega} e^{\int_{0}^{t} a_{2}(\tau) d \tau} x(t)=x(l) e^{\int_{0}^{l} a_{2}(\tau) d \tau} .
\end{gathered}
$$

We have

$$
\gamma(x)=\theta(x) \leq \alpha(x), \quad x \in P,
$$

and

(1.6) $\quad \gamma(x)=x(\eta) e^{\int_{0}^{\eta} a_{1}(\tau) d \tau} \geq e^{\int_{0}^{\eta} a_{1}(\tau) d \tau} \delta\|x\| \quad$ for each $x \in P$,

$$
\alpha(x)=x(l) e^{\int_{0}^{l} a_{2}(\tau) d \tau} \geq e^{\int_{0}^{l} a_{2}(\tau) d \tau} \delta\|x\| \quad \text { for each } x \in P .
$$

Then

$$
\begin{gathered}
\|x\| \leq e^{-\int_{0}^{\eta} a_{1}(\tau) d \tau} \frac{1}{\delta} \gamma(x)=e^{-\int_{0}^{\eta} a_{1}(\tau) d \tau} \frac{1}{\delta} \theta(x) \quad \text { for each } x \in P, \\
\|x\| \leq e^{-\int_{0}^{l} a_{2}(\tau) d \tau} \frac{1}{\delta} \alpha(x) \quad \text { for each } x \in P \\
\theta(\lambda x)=\lambda \theta(x) \quad \text { for all } \lambda \in[0,1] \text { and } x \in P .
\end{gathered}
$$

For the notational convenience, we set

$$
\begin{array}{ll}
\sigma_{1}=\frac{M_{1}(\omega-\eta)}{k_{2}-1} e^{\int_{0}^{\eta} a_{1}(\tau) d \tau}, & \varrho_{1}=\frac{M_{2} \omega}{k_{1}-1} e^{\int_{0}^{\eta} a_{1}(\tau) d \tau}, \\
\sigma_{2}=\frac{M_{1}(\omega-l)}{k_{2}-1} e^{\int_{0}^{l} a_{2}(\tau) d \tau}, & \varrho_{2}=\frac{M_{2} \omega}{k_{1}-1} e^{\int_{0}^{l} a_{2}(\tau) d \tau .}
\end{array}
$$

TheOREM 2.1. Suppose that there are positive numbers $a<b<c$ such that

$$
0<a<\frac{\sigma_{2}}{\varrho_{1}} b<\frac{\sigma_{2} \delta}{\varrho_{1}} c .
$$


Assume $f(t, \varphi)$ satisfies the following conditions:

(A) $f(t, \varphi)>c / \sigma_{1}$ for $(t, \varphi) \in[\eta, \omega] \times K_{1}$,

(B) $f(t, \varphi)<b / \varrho_{1}$ for $(t, \varphi) \in[0, \omega] \times K_{2}$,

(C) $f(t, \varphi)>a / \sigma_{2}$ for $(t, \varphi) \in[l, \omega] \times K_{3}$,

where

$$
\begin{aligned}
& K_{1}=\left\{\varphi \in C^{+}: c e^{-\int_{0}^{\eta} a_{1}(\tau) d \tau} \leq\|\varphi\|_{C} \leq(c / \delta) e^{-\int_{0}^{\eta} a_{1}(\tau) d \tau}\right\}, \\
& K_{2}=\left\{\varphi \in C^{+}: 0 \leq\|\varphi\|_{C} \leq(b / \delta) e^{-\int_{0}^{\eta} a_{1}(\tau) d \tau}\right\}, \\
& K_{3}=\left\{\varphi \in C^{+}: a e^{-\int_{0}^{l} a_{2}(\tau) d \tau} \leq\|\varphi\|_{C} \leq(a / \delta) e^{-\int_{0}^{l} a_{2}(\tau) d \tau}\right\} .
\end{aligned}
$$

Then (1.1) has at least two positive periodic solutions $x_{1}$ and $x_{2}$ satisfying

$$
a<\alpha\left(x_{1}\right), \quad \theta\left(x_{1}\right)<b, \quad b<\theta\left(x_{2}\right), \quad \gamma\left(x_{2}\right)<c .
$$

Proof. By Lemma 1.1, $T: \overline{P(\gamma, c)} \rightarrow P$ and $T$ is completely continuous. Now, we show that (i)-(iii) of Lemma 1.2 are satisfied.

First, we verify that $x \in \partial P(\gamma, c)$ implies $\gamma(T x)>c$. Since $\gamma(x)=$ $x(\eta) e^{\int_{0}^{\eta} a_{1}(\tau) d \tau}=c$, one gets

$$
x(t) e^{\int_{0}^{t} a_{1}(\tau) d \tau} \geq c \quad \text { for } t \in[\eta, \omega] .
$$

From (1.8), we have

$$
c e^{-\int_{0}^{\eta} a_{1}(\tau) d \tau} \leq\left\|x_{t}\right\|_{C} \leq e^{-\int_{0}^{\eta} a_{1}(\tau) d \tau} \frac{c}{\delta} \quad \text { for } t \in[\eta, \omega] .
$$

Then we get

$$
\begin{aligned}
\gamma(T x) & =(T x)(\eta) e^{\int_{0}^{\eta} a_{1}(\tau) d \tau}=e^{\int_{0}^{\eta} a_{1}(\tau) d \tau} \int_{\eta}^{\eta+\omega} G(\eta, s) f\left(s, x_{s}\right) d s \\
& \geq e^{\int_{0}^{\eta} a_{1}(\tau) d \tau} \int_{\eta}^{\omega} G(\eta, s) f\left(s, x_{s}\right) d s \\
& >e^{\int_{0}^{\eta} a_{1}(\tau) d \tau} \int_{\eta}^{\omega} \frac{M_{1}}{k_{2}-1} \frac{c}{\sigma_{1}} d s=c .
\end{aligned}
$$

Secondly, we prove that $x \in \partial P(\theta, b)$ implies $\theta(T x)<b$. Since $\theta(x)=b$ implies $x(\eta) e^{\int_{0}^{\eta} a_{1}(\tau) d \tau}=b$, we have

$$
0 \leq x(t) e^{\int_{0}^{t} a_{1}(\tau) d \tau} \leq b \quad \text { for } t \in[0, \eta] .
$$

From (1.8), we have

$$
0 \leq\left\|x_{t}\right\|_{C} \leq \frac{b}{\delta} e^{-\int_{0}^{\eta} a_{1}(\tau) d \tau} \quad \text { for } t \in[0, \omega] \text { or } t \in[\eta, \eta+\omega] .
$$


Then

$$
\begin{aligned}
\theta(T x) & =(T x)(\eta) e^{\int_{0}^{\eta} a_{1}(\tau) d \tau}=e^{\int_{0}^{\eta} a_{1}(\tau) d \tau} \int_{\eta}^{\eta+\omega} G(\eta, s) f\left(s, x_{s}\right) d s \\
& \leq e^{\int_{0}^{\eta} a_{1}(\tau) d \tau} \int_{\eta}^{\eta+\omega} \frac{M_{2}}{k_{1}-1} \frac{b}{\varrho_{1}} d s=b .
\end{aligned}
$$

Finally, we show that

$$
P(\alpha, a) \neq \emptyset, \quad \alpha(T x)>a \quad \text { for all } x \in \partial P(\alpha, a) .
$$

The nonemptiness is obvious. On the other hand, $\alpha(x)=x(l) e^{\int_{0}^{l} a_{2}(\tau) d \tau}=a$ implies $a \leq x(t) e^{\int_{0}^{t} a_{2}(\tau) d \tau}$ for $t \in[l, \omega]$. Recalling (1.9), we know

$$
a e^{-\int_{0}^{l} a_{2}(\tau) d \tau} \leq\left\|x_{t}\right\|_{C} \leq e^{-\int_{0}^{l} a_{2}(\tau) d \tau} \frac{a}{\delta} \quad \text { for } t \in[l, \omega]
$$

Thus

$$
\begin{aligned}
\alpha(T x) & =(T x)(l) e^{\int_{0}^{l} a_{2}(\tau) d \tau}=e^{\int_{0}^{l} a_{2}(\tau) d \tau} \int_{l}^{l+\omega} G(l, s) f\left(s, x_{s}\right) d s \\
& \geq e^{\int_{0}^{l} a_{2}(\tau) d \tau} \int_{l}^{\omega} G(l, s) f\left(s, x_{s}\right) d s \\
& >(\omega-l) e^{\int_{0}^{l} a_{2}(\tau) d \tau} \frac{M_{1}}{k_{2}-1} \frac{a}{\sigma_{2}}=a .
\end{aligned}
$$

Thus by Lemma 1.2, $T$ has at least two different fixed points $x_{1}$ and $x_{2}$, which are positive periodic solutions of (1.1). The proof is complete.

Similarly, by Lemma 1.5, we have the following result.

THEOREM 2.2. Suppose that there are positive numbers $0<a<b<c$ such that

$$
0<a<\delta b<\sigma_{1} \delta c / \varrho_{1} .
$$

Assume $f(t, \varphi)$ satisfies the following conditions:

$$
\begin{aligned}
& \left(\mathrm{A}^{\prime}\right) f(t, \varphi)<c / \varrho_{1} \text { for }(t, \varphi) \in[0, \omega] \times K_{1}^{\prime}, \\
& \left(\mathrm{B}^{\prime}\right) f(t, \varphi)>b / \sigma_{1} \text { for }(t, \varphi) \in[\eta, \omega] \times K_{2}^{\prime}, \\
& \left(\mathrm{C}^{\prime}\right) f(t, \varphi)<a / \varrho_{2} \text { for }(t, \varphi) \in[0, \omega] \times K_{3}^{\prime},
\end{aligned}
$$

where

$$
\begin{aligned}
& K_{1}^{\prime}=\left\{\varphi \in C^{+}: 0 \leq\|\varphi\|_{C} \leq(c / \delta) e^{-\int_{0}^{\eta} a_{1}(\tau) d \tau}\right\}, \\
& K_{2}^{\prime}=\left\{\varphi \in C^{+}: b e^{-\int_{0}^{\eta} a_{1}(\tau) d \tau} \leq\|\varphi\|_{C} \leq(b / \delta) e^{-\int_{0}^{\eta} a_{1}(\tau) d \tau}\right\}, \\
& K_{3}^{\prime}=\left\{\varphi \in C^{+}: 0 \leq\|\varphi\|_{C} \leq(a / \delta) e^{-\int_{0}^{l} a_{2}(\tau) d \tau}\right\} .
\end{aligned}
$$

Then (1.1) has at least three positive solutions $x_{1}, x_{2}$ and $x_{3}$ satisfying

$$
a<\alpha\left(x_{1}\right), \quad \theta\left(x_{1}\right)<b, \quad b<\theta\left(x_{2}\right), \quad \gamma\left(x_{2}\right)<c .
$$


The proof is omitted since it is similar to that of Theorem 2.1.

Now, we give theorems which may be considered as corollaries of Theorems 2.1 and 2.2 .

Choose $\varepsilon_{1}, \varepsilon_{2}, \varepsilon_{3}$ such that

$$
\begin{gathered}
\varepsilon_{1} \sigma_{2} e^{-\int_{0}^{l} a_{2}(\tau) d \tau}>1, \quad \varepsilon_{2} \sigma_{1} e^{-\int_{0}^{\eta} a_{1}(\tau) d \tau}>1, \\
0<\varepsilon_{3} \frac{\max \left\{\varrho_{1}, \varrho_{2}\right\}}{\delta} e^{-\int_{0}^{\eta} a_{1}(\tau) d \tau}<1 .
\end{gathered}
$$

THEOREM 2.3. Let the following conditions be satisfied:

(D) $\lim _{\|\varphi\|_{C} \rightarrow 0} \min _{t \in[l, \omega]} \frac{f(t, \varphi)}{\|\varphi\|_{C}}>\varepsilon_{1} ; \lim _{\|\varphi\|_{C} \rightarrow \infty} \min _{t \in[\eta, \omega]} \frac{f(t, \varphi)}{\|\varphi\|_{C}}>\varepsilon_{2}$;

(E) there exists a $p_{1}>0$ such that for each $\varphi$ with $0 \leq\|\varphi\|_{C} \leq$ $\left(p_{1} / \delta\right) e^{-\int_{0}^{\eta} a_{1}(\tau) d \tau}$

$$
f(t, \varphi)<p_{1} / \varrho_{1} \quad \text { for all } t \in[0, \omega] .
$$

Then (1.1) has at least two positive periodic solutions.

Proof. First, choose $b=p_{1}$; one gets

$$
f(t, \varphi)<\frac{p_{1}}{\varrho_{1}}=\frac{b}{\varrho_{1}} \quad \text { for } t \in[0, \omega], 0 \leq\|\varphi\|_{C} \leq \frac{b}{\delta} e^{-\int_{0}^{\eta} a_{1}(\tau) d \tau} .
$$

Secondly, since

$$
\lim _{\|\varphi\|_{C} \rightarrow 0} \min _{t \in[l, \omega]} \frac{f(t, \varphi)}{\|\varphi\|_{C}}>\varepsilon_{1}
$$

there is $R_{1}>0$ sufficiently small such that

$$
f(t, \varphi)>\varepsilon_{1}\|\varphi\|_{C} \quad \text { for } t \in[l, \omega], 0 \leq\|\varphi\|_{C} \leq R_{1} .
$$

Without loss of generality, suppose

$$
R_{1} \leq \frac{\sigma_{2}}{\varrho_{1} \delta} b e^{-\int_{0}^{l} a_{2}(\tau) d \tau} .
$$

Choose $a>0$ so that $a<\delta R_{1} e^{\int_{0}^{l} a_{2}(\tau) d \tau}$. For

$$
a e^{-\int_{0}^{l} a_{2}(\tau) d \tau} \leq\|\varphi\|_{C} \leq e^{-\int_{0}^{l} a_{2}(\tau) d \tau} \frac{a}{\delta},
$$

we have $\|\varphi\|_{C} \leq R_{1}$ and $a<\left(\sigma_{2} / \varrho_{1}\right) b$. Thus

$$
\begin{aligned}
& f(t, \varphi)>\varepsilon_{1}\|\varphi\|_{C} \geq \varepsilon_{1} a e^{-\int_{0}^{l} a_{2}(\tau) d \tau}>a / \sigma_{2} \\
& \quad \text { for } t \in[l, \omega], a e^{-\int_{0}^{l} a_{2}(\tau) d \tau} \leq\|\varphi\|_{C} \leq e^{-\int_{0}^{l} a_{2}(\tau) d \tau} \frac{a}{\delta} .
\end{aligned}
$$

Thirdly, since

$$
\lim _{\|\varphi\|_{C} \rightarrow \infty} \min _{t \in[\eta, \omega]} \frac{f(t, \varphi)}{\|\varphi\|_{C}}>\varepsilon_{2},
$$

there is $R_{2}>0$ sufficiently large such that

$$
f(t, \varphi)>\varepsilon_{2}\|\varphi\|_{C} \quad \text { for } t \in[\eta, \omega],\|\varphi\|_{C} \geq R_{2} .
$$


Without loss of generality, suppose $R_{2}>(b / \delta) e^{-\int_{0}^{\eta} a_{1}(\tau) d \tau}$. Choose $c \geq$ $R_{2} e^{\int_{0}^{\eta} a_{1}(\tau) d \tau}$. Then

$$
\begin{aligned}
f(t, \varphi)>\varepsilon_{2}\|\varphi\|_{C} & \geq \varepsilon_{2} c e^{-\int_{0}^{\eta} a_{1}(\tau) d \tau}>c / \sigma_{1} \\
& \text { for } t \in[\eta, \omega], c e^{-\int_{0}^{\eta} a_{1}(\tau) d \tau} \leq\|\varphi\|_{C} \leq e^{-\int_{0}^{\eta} a_{1}(\tau) d \tau} \frac{c}{\delta} .
\end{aligned}
$$

We now get $0<a<\sigma_{2} b / \varrho_{1}<\sigma_{2} \delta c / \varrho_{1}$, and then the conditions in Theorem 2.1 are all satisfied. By Theorem 2.1, (1.1) has at least two positive periodic solutions. The proof is complete.

THEOREM 2.4. Let the following conditions be satisfied:

(F) $\lim _{\|\varphi\|_{C} \rightarrow 0} \max _{t \in[0, \omega]} \frac{f(t, \varphi)}{\|\varphi\|_{C}}<\varepsilon_{3}$;

(G) there exists a $p_{2}>0$ such that for each $\varphi$ with $p_{2} e^{-\int_{0}^{\eta} a_{1}(\tau) d \tau} \leq$ $\|\varphi\|_{C} \leq\left(p_{2} / \delta\right) e^{-\int_{0}^{\eta} a_{1}(\tau) d \tau}$

$$
f(t, \varphi)>p_{2} / \sigma_{1} \quad \text { for all } t \in[\eta, \omega] .
$$

Then (1.1) has at least three positive periodic solutions.

The following corollaries are obvious.

Corollary 2.1. Let the following conditions be satisfied:

$\left(\mathrm{D}^{\prime}\right) \lim _{\|\varphi\|_{C} \rightarrow 0} \min _{t \in[l, \omega]} \frac{f(t, \varphi)}{\|\varphi\|_{C}}=\infty ; \lim _{\|\varphi\|_{C} \rightarrow \infty} \min _{t \in[\eta, \omega]} \frac{f(t, \varphi)}{\|\varphi\|_{C}}=\infty ;$

(E) there exists a $p_{1}>0$ such that for each $\varphi$ with $0 \leq\|\varphi\|_{C} \leq$ $\left(p_{1} / \delta\right) e^{-\int_{0}^{\eta} a_{1}(\tau) d \tau}$

$$
f(t, \varphi)<p_{1} / \varrho_{1} \quad \text { for all } t \in[0, \omega] .
$$

Then (1.1) has at least two positive periodic solutions.

Corollary 2.2. Let the following conditions be satisfied:

$(\mathrm{F})^{\prime} \lim _{\|\varphi\|_{C} \rightarrow 0} \max _{t \in[0, \omega]} \frac{f(t, \varphi)}{\|\varphi\|_{C}}=0 ;$

(G) there exists a $p_{2}>0$ such that for each $\varphi$ with $p_{2} e^{-\int_{0}^{\eta} a_{1}(\tau) d \tau} \leq$ $\|\varphi\|_{C} \leq\left(p_{2} / \delta\right) e^{-\int_{0}^{\eta} a_{1}(\tau) d \tau}$

$$
f(t, \varphi)>p_{2} / \sigma_{1} \quad \text { for all } t \in[\eta, \omega] .
$$

Then (1.1) has at least three positive periodic solutions.

\section{References}

[1] R. I. Avery, C. J. Chyan and J. Henderson, Twin positive solutions of boundary value problems for ordinary differential equations and finite difference equations, Comput. Math. Appl. 42 (2001), 695-704. 
[2] S. S. Cheng and G. Zhang, Existence of positive periodic solutions for non-autonomous functional differential equations, Electron. J. Differential Equations 2001, no. 59,8 pp.

[3] D. Jiang and J. Wei, Existence of positive periodic solutions for Volterra integrodifferential equations, Acta Math. Sci. Ser. B 21 (2001), 553-560.

[4] D. Q. Jiang, J. Wei and B. Zhang, Positive periodic solutions for functional differential equations and population models, Electron. J. Differential Equations 2002, no. $71,13 \mathrm{pp}$.

[5] M. A. Krasnosel'skiı̌, Positive Solutions of Operator Equations, Noordhoff, Groningen, 1964.

[6] Z. Teng and L. Chen, Global asymptotic stability of periodic Lotka-Volterra systems with delays, Nonlinear Anal. 45 (2001), 1081-1095.

School of Applied Mathematics

Guangdong University of Technology

Guangzhou 510006, P.R. China

E-mail: scx168@sohu.com

Received 6.7.2007

and in final form 22.11.2007 